\title{
Market Power and Efficiency in Card Payment Systems: A Comment
}

\author{
LUIS M. B. CABRAL * \\ New York University and CEPR
}

\begin{abstract}
In this short comment, I provide my views on "Market Power and Efficiency in Card Payment Systems" (published in this issue) that was presented at the Antitrust Activity in Card-Based Payment Systems: Causes and Consequences conference, and more generally on the ideas introduced by Rochet and Tirole's work on payment systems.
\end{abstract}

\section{Introduction}

Beginning with their seminal 2002 paper, Rochet and Tirole have contributed to the economic analysis of card payments systems with a series of research papers $(2003,2004$, 2005a, 2005b). ${ }^{1}$ In this paper, I comment on the main ideas that run through this series of papers, which I will collectively refer to by the authors' initials, RT.

In Section 2, I lay down a simple basic model that essentially captures the features of the RT models. In Section 3, I propose a set of assumptions that best captures the scenario envisaged by RT, and derive a set of results that reflect the essence of their analysis. I then explore, in Sections 4 and 5, the possibility of buyer and seller heterogeneity. Although RT do consider this possibility, they do not develop explicit results as I do here. Section 6 summarizes the analysis in this paper.

I show that if usage externalities are present then it is possible for the equilibrium with market power to yield higher social surplus than the competitive equilibrium. Although card fees are higher with market power than with competition, the structure of card fees is more efficient under market power. Specifically, market power allows for the internalization of usage externalities in card payments. To a great extent, this is the main point in RT's analysis.

If there is buyer or seller heterogeneity, however, then market power has a negative effect. Departures from marginal cost pricing lead to Harberger-triangle-type welfare losses. These Harberger triangles correspond to optimal sales that do not take place in equilibrium because of market power (case of buyer heterogeneity); or sales that are paid with cash when it would be socially efficient to pay by card (case of seller heterogeneity).

\footnotetext{
* Department of Economics, Stern School of Business New York University 44 West 4th Street, New York, NY 10012-1126 E-mail: lcabral@,stern.nyu.edu

${ }^{1}$ See also Rochet (2003).
} 
In other words, while RT are correct in stressing that two-sided markets are unlike other markets, it still remains the case that departures from marginal cost pricing have potentially negative welfare effects.

\section{Basic model}

Throughout the paper, I consider a game with a timing structure identical to RT. Specifically, the card payment system is modeled as a game with three stages:

(1) Network operators (issuers, acquirers, card association) determine buyer, merchant and interchange fees.

(2) The seller decides whether to accept cards and sets sale price.

(3) The buyer decides whether to buy and which method of payment to use (if there is a choice).

As much as possible, I will also follow RT's notation. The buyer and merchant fees are denoted $p_{B}$ and $p_{S}$, respectively. That is, for each card transaction the buyer pays $p_{B}$ (possibly a negative amount) and the seller pays $p_{S .}{ }^{2}$ The buyer's benefit from paying with a card (gross of card fees) is given by $b_{B}$. That is, by paying with a card the buyer's benefit is $b_{B}$ higher than by paying with cash. ${ }^{3}$ Let $b_{S}$ be the corresponding benefit for the seller.

For simplicity, I will assume that production costs and network costs are zero. (Alternatively, one may interpret the values that follow as markups over constant marginal costs.) The retail price set by the seller is $p$. Finally, let $u$ be the buyer's willingness to pay for the seller's product. (Later on I will allow for heterogeneity in the values of $u$ and $b$.)

Throughout the paper, I will maintain the following assumption regarding parameter values:

Assumption 1: $u>0$ and $b_{B}+b_{S}>0$.

Together, these assumptions imply that it is socially optimal for a sale to occur and for payment to be made with a card. In the next few sections, I will consider a variety of possible inefficiencies; these will either take the form of foregone sales or sales that are made without the use of a card.

I consider two possible extreme structures. At one extreme, both issuing and acquiring are competitive, and the card association is not for profit, so that card fees are zero (for both the buyer and the seller). At the opposite extreme, a monopoly firm controls issuing, acquiring, and the card association. Although the perfect competition and monopoly cases do not occur in practice, I believe they provide a good first-order approximation to the reality of a number of countries. ${ }^{4}$

\footnotetext{
${ }^{2}$ For simplicity, and again following RT, I assume that all transactions are of the same size; and with no further loss of generality I normalize transaction size to 1 .

${ }^{3}$ For simplicity, I assume cash in the best alternative to card payments.

${ }^{4}$ I should mention that, in addition to these two extremes, RT also consider a third market structure, namely a not-for-profit monopoly network; see, for example, Rochet and Tirole (2002). Insofar as I am mainly
} 
Given these two extreme network structures, we need not focus on interchange fees per se. In the competitive case, interchange fees are zero, whereas in the monopoly case they are a simple transfer price. In other words, all the relevant information is summarized in the buyer and merchant fees ( $p_{B}$ and $p_{S}$, respectively).

In the next three sections I consider three particular versions of the basic model. These correspond to different values of the main parameters. Each example illustrates one of several important effects in the context of payment card networks. The first example (Section 3) illustrates the central point in RT, namely that a network with market power may achieve higher social welfare than a competitive network or one that is subject to costbased regulation. Section 4 considers the case of heterogeneity in buyer valuations. I show that, under reasonable circumstances, monopoly supply leads to higher merchant fees than competition, and this in turn leads to lower sales than optimal. Finally, Section 5 considers the case of heterogeneity in seller benefit from card usage. I show that, again under reasonable circumstances, monopoly supply leads to higher merchant fees than competition, and this in turn leads to lower card usage than optimal.

\section{Usage externality}

The first scenario I consider reflects the central point in RT, namely that in two-sided markets such as card payment systems there are important usage externalaties; and that a network system with market power may internalize these externalities better than a competitive market structure. In order to make this point, I specialize the general model introduced in the previous section by assuming that

Assumption 2: $b_{B}<0$ and $b_{S}>0$.

Assumption 2 is the best case for the inefficiency of a competitive equilibrium in twosided markets. The point is that consumers have no incentive to use cards even though it is socially optimal to do so. In RT terms, there is a usage externality. Formally, we have the following result. (All proofs are included in the Appendix.)

Proposition 1: Suppose Assumption 2 holds. Under competitive card supply, there exists a unique subgame perfect Nash equilibrium: card fees are zero; the seller accepts cards and sets a price $p=u$; the buyer makes a purchase and pays with cash. Finally, social welfare is suboptimal.

When usage externalities are present, it is quite possible that a network system with market power perform better than a competitive one. The reason is that a monopoly network (to consider the extreme situation) is better able to internalize the usage externality by creating the appropriate fee structure. In the present case, this consists of subsidizing the buyer so that it chooses card payments, as social optimality would prescribe. Formally,

interested in illustrating possibility results, it suffices for me to consider the extreme cases. From an applied point of view, the question is then how close each particular situation is to the extremes considered here and in the RT papers. 
Proposition 2: Suppose Assumption 2 holds. Under monopoly card supply, there exists an essentially unique subgame perfect Nash equilibrium: card fees are $p_{B}=b_{B}, p_{S}=b_{S}$; the seller accepts cards and sets a price $p=u$; the buyer makes a purchase and pays with card. Finally, social welfare is optimal.

The qualification "essentially" means that, although there are several subgame perfect Nash equilibria, they all imply the same level of consumer surplus, seller profit, network profit and social welfare.

Proposition 2 is an important result, and it is worth exploring its main intuition. Suppose card payments are used in equilibrium. Then the buyer is willing to pay $u+b_{B}$ $p_{B}$. If the seller charges a price equal to the buyer's willingness to pay, then its profit is given by

(1) $\pi=u+b_{B}-p_{B}+b_{S}-p_{\mathrm{S}}$.

The seller's alternative is to reject card payments. Then the buyer's willingness to pay is simply $u$, and so is seller's profit:

(2) $\pi=u$.

Equating (1) to (2), we get the network's maximum $p_{S}$ such that the seller is still willing to accept card payments. It is given by

$p_{S}=b_{B}+b_{S}-p_{B}$

It follows that the network's profit is given by

$p_{S}+p_{B}=b_{B}+b_{S}$.

This is an important equation, for it implies that the network's profit is positive if and only if the total social benefit from card usage is positive. If $b_{B}+b_{S}$ is positive, then a monopoly network will set a fee structure such that card payments are used, as efficiency dictates. If, on the other hand, $b_{B}+b_{S}$ is negative, then a monopoly network cannot make a positive profit from card usage, and so cards are not used in equilibrium, as efficiency dictates.

The intuition for the idea that market power may imply higher efficiency can be summarized in two simple catchy phrases: "It takes two to tango" and "What goes around comes around." Suppose one side of the market receives a negative benefit from a match but the other side receives a value that is positive and greater in absolute value. Then subsidizing the side that receives a negative value may increase social welfare. A competitive issuer would have no incentive to provide such subsidy. But a monopoly card supplier does. In fact, the monopolist knows that an extra dollar given to the card user will be reflected in an extra dollar of sale price and thus an extra dollar in merchant's willingness to pay for card acceptance.

In summary, market power in card supply has the benefit of allowing fees to be set taking into account the two-sidedness of a card payment system. Notice that in the simple model we considered so far there is no uncertainty about buyer or seller valuations. (Or 
equivalently, in a situation with many buyers and sellers, there is no buyer or seller heterogeneity.) This assumption implies that there are no distortions in price setting in terms of purchase or card acceptance decisions. Therefore, the possibility that the level of fees set by the card system be very high has no other consequence than a transfer from merchants to the card network; ${ }^{5}$ it has no efficiency implications (that is, no Harberger triangles are created). In the next two sections, I will consider the possibility of buyer and seller heterogeneity and show that market power may have negative efficiency implications.

\section{$4 \quad$ Buyer heterogeneity}

In this section, I consider the possibility of uncertainty about the buyer's valuation. (In a situation with many buyers, this corresponds to heterogeneity in buyer valuations.) Specifically, I continue to assume that there is one seller with zero production cost; but now I assume there are two types of buyer: a fraction $1 / 2$ is willing to pay up to $u^{H}$; and a fraction $1 / 2$ is willing to pay only $u^{L}<u^{H}$. I make the following assumption regarding buyer and seller valuations:

Assumption 3: $b_{B}>0, b_{S}>0$ and $0<u^{H}-2 u^{L}<b_{B}+b_{S}$.

Since both $b_{B}$ and $b_{S}$ are positive, there is no usage externality (in the previous section we had a usage externality because $b_{B}<0$ ). I will next show that, since there is no usage externality, market power no longer has the benefit considered in the previous section. Moreover, given buyer heterogeneity, market power may imply retail price inefficiencies (Harberger triangles). I first show that, under Assumption 3, the competitive equilibrium is efficient.

Proposition 3: Suppose Assumption 3 holds. Under competitive card supply, there exists a unique subgame perfect Nash equilibrium: card fees are zero; the seller accepts cards and sets a price $p=u^{L}+b_{B}$; and both buyer types use a card and make a purchase. Finally, the solution is socially optimal.

As in the previous section, I now consider the case of monopoly card supply.

Proposition 4: Suppose Assumption 3 holds. Under monopoly card supply, there exists an essentially unique subgame perfect Nash equilibrium: total card fees are $p_{B}+p_{S}=b_{B}+b_{S}$; the seller accepts cards and sets a price $p=u^{H}+b_{B}-p_{B}$; high buyer types use a card and make a purchase; low buyer types don't make any purchase. Finally, the solution is socially suboptimal.

The source of inefficiency under buyer heterogeneity and monopoly card supply is that market power at the network level translates into high merchant fees which in turn are

\footnotetext{
${ }^{5}$ That is, starting from the equilibrium in Proposition 2, if the network were to reduce the overall level of fees it would do so by reducing $p_{S}$. The overall level of efficiency would remain the same; there would simply be a transfer from the card network to merchants.
} 
(partially) passed on to consumers. Since consumer demand is elastic, a higher seller cost leads to allocative inefficiency, namely fewer sales than socially optimal.

\section{$5 \quad$ Seller heterogeneity}

In this section, I consider the possibility of heterogeneity in seller benefit from card usage. Specifically, suppose that seller benefit may be low, $b_{S}^{L}$, or high, $b_{S}^{H}$. Moreover, suppose the following relation between these parameters holds:

Assumption 4: $b_{B}>0, b_{S}^{i}>0(i=L, H)$, and $0<b_{S}^{L}<\left(b_{S}^{H}-b_{B}\right) / 2$

Since I continue to assume that both buyer and seller receive a positive benefit from card usage, the externality identified in Section 2 is not present. In fact, as in the previous section, the equilibrium under competitive card supply is socially efficient.

Proposition 5: Suppose Assumption 4 holds. Under competitive card supply, there exists a unique subgame perfect Nash equilibrium: card fees are zero; both types of seller accept card payments and set price $p=u+b_{B}$; and the buyer makes a purchase. Finally, the equilibrium is socially optimal.

As in the previous section, I now show that market power in card supply does introduce inefficiencies.

Proposition 6: Suppose Assumption 4 holds. Under monopoly card supply, there exists an essentially unique subgame perfect Nash equilibrium: total card fees are $p_{B}+p_{S}=b_{B}+$ $b_{S}^{H}$; cards are accepted only by high-type sellers, who set a price $p=u+b_{B}-p_{B}$; cards are not accepted by low-type sellers, who set a price $p=u$. Finally, the solution is socially suboptimal.

In Proposition 4, the source of inefficiency is buyer heterogeneity: the seller must decide whether to price for all types or for high types only. And merchant fees induce the seller to switch from targeting all types to targeting high types only. In Proposition 6, we now have a different source of heterogeneity, seller heterogeneity. Now it's the network (specifically, acquirers) that must decide whether to target all types of seller or simply high types. Under competitive card supply fees are zero, and so both types of seller accept cards. Under monopoly supply, however, and assuming Assumption 4 holds, the network finds it optimal to target high types only, thus creating a "Harberger triangle" or inefficiency. 


\begin{tabular}{|l|c|c|c|}
\hline \multirow{4}{*}{} & \multicolumn{3}{|c|}{ Scenario } \\
\cline { 2 - 4 } & $\begin{array}{c}\text { A } \\
\text { Usage } \\
\text { externality }\end{array}$ & $\begin{array}{c}\text { B } \\
\text { Buyer } \\
\text { heterogeneity }\end{array}$ & $\begin{array}{c}\text { C } \\
\text { Seller } \\
\text { heterogeneity }\end{array}$ \\
\hline \hline \multicolumn{4}{|c|}{ Competition } \\
\hline Structure of fees & suboptimal & OK & OK \\
\hline Size of fees & OK & OK & OK \\
\hline Welfare & suboptimal & optimal & optimal \\
\hline \hline \multicolumn{4}{|c|}{ Monopoly } \\
\hline Structure of fees & OK & OK & OK \\
\hline Size of fees & OK & excessive & excessive \\
\hline Welfare & optimal & suboptimal & suboptimal \\
\hline
\end{tabular}

Table 1: Summary of Main Results

\section{Discussion}

A summary of the main results in Sections 3-5 in presented in Table 1. Each column in the table corresponds to each of the scenarios I considered: usage externality, buyer heterogeneity, seller heterogeneity. For each scenario, I considered two extreme cases: perfect competition and monopoly. The cases correspond to two sets of rows. In each set, I indicate whether the structure of fees, the level of fees, or the level of social welfare are optimal or suboptimal.

I define the level or structure of fees to be OK if social welfare cannot be increased by changing only the level or the structure of fees, respectively. The structure of fees is suboptimal if social welfare can be increased by changing the structure of fees while maintaining the level. The level of fees is excessive if social welfare can be increased by decreasing the level of fees while maintaining the structure.

Table 1 indicates that, under Scenario A (usage externality), the equilibrium with market power is welfare superior to the competitive equilibrium. This is one of the situations analyzed by RT. The problem with the competitive equilibrium is that it leads to an inefficient structure of card fees. The advantage of a card system with market power is that it has the right incentives to internalize the usage externality. Since there is no buyer or seller heterogeneity, higher fees have no welfare effect: they are simply a transfer from sellers to the card network.

Under Scenarios B and C, since both buyer and seller have positive valuations from card use, no usage externality takes place. It follows that the benefits from market power are absent. Moreover, since there is buyer heterogeneity (Scenario B) and seller heterogeneity (Scenario C) market power implies an inefficient allocation of resources. It follows that in both Scenarios B and $\mathrm{C}$ the competitive solution is superior from a social welfare point of view. 


\section{Conclusion}

Rochet and Tirole make the valid point that two-sided networks are complex systems. In particular, one cannot think of interchange fees simply as a fee for a service; interchange fees also help balancing usage externalities in card payment systems. Although I agree with this point, I would also add that Econ 1 still applies. In particular, departures from marginal cost pricing are bound to lead to inefficient distortions: Harberger triangles exist.

Rochet and Tirole argue convincingly against cost-based regulation of card payment systems. I agree that simple-minded cost regulation would be just that - simple-minded. But the observation of departures from marginal cost pricing suggest that there may be important welfare losses; and that there may be a rationale for regulation.

\section{$8 \quad$ References}

Rochet, J.C. and J. Tirole (2002) "Cooperation Among Competitors: Some Economics of Payment Card Associations," Rand Journal of Economics, 33: 1-22.

Rochet, J.C. and J. Tirole (2003) "Platform Competition in Two-Sided Markets," Journal of the European Economic Association, 1: 990-1029.

Rochet, J.C. and J. Tirole (2004) “Two-Sided Markets: An Overview,” mimeo, IDEI.

Rochet, J.C. and J. Tirole (2005a) "A Primer on Payment Cards," Report Prepared for the Portuguese Competition Authority.

Rochet, J.C. and J. Tirole (2005b) "The Welfare Impact of Interchange Fees," mimeo, IDEI.

Rochet, J.C. (2003) "The Theory of Interchange Fees: A Synthesis of Recent Contributions," Review of Network Economics, 2: 97-124.

\section{$9 \quad$ Appendix}

Proof of Proposition 1: If the credit card system is competitive, neither consumers nor merchants pay card fees. Since $b_{B}<0$, the buyer prefers to pay cash. It follows that the buyer's willingness to pay is $u$, which is therefore the price set by the seller.

Proof of Proposition 2: In order to induce buyers to use cards, the network must set $p_{B} \leq b_{B}$. Suppose specifically that $p_{B}=b_{B}$. Then the buyer is willing to pay $u$ either in cash or with card. It follows that the seller optimally charges $u$. If the seller accepts cards, then it receives a profit of

$\pi=u+b_{S}-p_{\mathrm{S}}$ 
If, on the other hand, the seller rejects credit cards, then its profit is given by $u$. It follows that the network's optimal merchant fee is $p_{S}=b_{S}$. Finally, the network's profit is given by $b_{B}+b_{S}$, which, by Assumption 2, is positive.

Proof of Proposition 3: If the credit card system is competitive, neither consumers nor merchants pay card fees. Since $b_{B}>0$ and $b_{S}>0$, it follows that credit cards are accepted and used. The seller has essentially two price levels it can set. If $p=u^{L}+b_{B}$, then both types of seller buy and seller's profit is given by

(3) $\pi=u^{L}+b_{B}+b_{\mathrm{S}}$

If, on the other hand, the seller sets $p=u^{H}+b_{B}$, then only high-type buyers buy, in which case profit is given by

(4) $\pi=\left(u^{H}+b_{B}+b_{\mathrm{S}}\right) / 2$.

Putting (3) and (4) together, the condition that it is optimal to sell to both buyer types reduces to

$u^{H}-2 u^{L}<b_{B}+b_{\mathrm{S}}$

which is true by assumption.

Proof of Proposition 4: Buyers are willing to pay $u+b_{B}-p_{B}$ with card or $u$ without card, where $u=u^{H}$ or $u^{L}$ depending on buyer type. Conditional on accepting cards, a seller is better off selling only to high consumer types if and only if

$\left(u^{H}+b_{B}-p_{B}+b_{S}-p_{\mathrm{S}}\right) / 2>u^{L}+b_{B}-p_{B}+b_{S}-p_{\mathrm{S}}$,

or simply

(5) $u^{H}-2 u^{L}>b_{B}+b_{\mathrm{S}}-p_{B}-p_{\mathrm{S}}$.

Notice that (5) depends only on the sum $p_{B}+p_{S}$ and so does the credit card network profit. I will therefore assume the value of $p_{B}$ is chosen so that buyers prefer to use cards with respect to cash. (Otherwise, buyers would choose cash and the system would make zero profits.) The seller has the option of refusing to accept cards. Conditional on refusing card payments, the seller is better off by selling only to high types if and only if

$0<u^{H}-2 u^{L}$,

which is true by assumption. The seller's option value in case it refuses credit cards is therefore $u^{H} / 2$. Given this option value, and given that the seller sells exclusively to high types when accepting credit cards, the network's optimal fees are such that the seller receives a profit equal to the option value of refusing credit cards. We thus have

$\left(u^{H}+b_{B}-p_{B}+b_{S}-p_{\mathrm{S}}\right) / 2=u^{H} / 2$, 
so simply

$p_{B}+p_{\mathrm{S}}=b_{B}+b_{\mathrm{S}}$

Notice that, at this fee level, $u^{H}-2 u^{L}>b_{B}+b_{S}-p_{B}-p_{S}=0$, which is true by Assumption 3 , so the hypothesis that the seller sells only to high types is confirmed. Finally, since there are no coordination issues (for example, several sellers), it is straightforward to show that there can be no equilibrium in which the seller refuses cards: the card system would then be better off by offering terms such that the seller would accept cards.

Proof of Proposition 5: Competition implies $p_{B}=p_{S}=0$. The buyer is therefore willing to pay $u+b_{B}$. Both types of seller optimally accept cards (as they are costless) and set $p=u$ $+b_{B}$.

Proof of Proposition 6: Since there is no buyer heterogeneity, the seller's problem is relatively simple: either he accepts credit cards or he doesn't. In either case, he will price to extract all of the buyer's surplus. Therefore, if credit cards are accepted, then $p=u+b_{B}$ if credit cards are accepted, and $p=u$ if credit cards are not accepted. It follows that seller's profit, if credit cards are accepted, is given by

(6) $\pi=u+b_{B}-p_{B}+b_{S}-p_{\mathrm{S}}$,

where $b_{S}=b_{S}^{L}$ or $b_{S}^{H}$; and if credit cards are not accepted then

(7) $\pi=u$.

The card system will set $p_{B}$ and $p_{S}$ such that one of the types of seller is just indifferent between accepting and not accepting cards. Equating the values of $\pi$ in (6) and (7) we get

$b_{B}-p_{B}+b_{S}-p_{\mathrm{S}}=0$,

where $b_{S}=b_{S}^{L}$ or $b_{S}^{H}$. Once again, notice that only the total fees $p_{B}+p_{S}$ matter. I will therefore assume the value of $p_{B}$ is chosen so that buyers prefer to use cards with respect to cash. (Otherwise, buyers would choose cash and the system would make zero profits.)

The condition that the card system prefers to exclude low benefit sellers $\left(b_{S}^{L}\right)$ is that profit from targeting both sellers is lower than targeting high-type sellers only. Targeting both types of seller requires $p_{B}+p_{\mathrm{S}}=b_{B}+b_{S}^{L}$, whereas targeting high-type sellers allows for a higher total fee, namely $p_{B}+p_{\mathrm{S}}=b_{B}+b_{S}^{H}$. We thus have the condition 
$\left(b_{B}+b_{S}^{H}\right) / 2>b_{B}+b_{S}^{L}$,

or simply

$\left(b_{S}^{H}-b_{B}\right) / 2>b_{S}^{L}$,

which is true by assumption. 